\title{
COMPARATIVE STUDY OF SCALES AND CONSISTENCY MEASURES IN THE AHP
}

\author{
Victoria Kusherbaeva ${ }^{1}$, Yuriy Sushkov ${ }^{2}$, Gayk Tamazyan ${ }^{3} *$ \\ Department of Mathematics \& Mechanics \\ Saint-Petersburg State University \\ St. Petersburg, Russia \\ E-mail1: kusher.v@gmail.com \\ Email²: Yu.Sushkov@mail.ru \\ Email $^{3}$ : tamazyangayk@gmail.com
}

\begin{abstract}
In this paper we research the analytic hierarchy process from the aspect of scale validity. Parameterization of scales is introduced and the properties of parameters are considered. Alternative scales to the classical one are investigated. The scales are compared to each other via two simulation studies. Two consistency measures are presented and interconnection between them is shown. Finally, recommendations to a decision maker are given.
\end{abstract}

Keywords: pairwise comparisons, scales, inconsistency, circular triads, logistic scale

\section{Introduction}

One of the essential points of the analytic hierarchy process is mapping qualitative estimates of pairwise comparisons into numerical ones. This mapping is called a scale. The fundamental scale proposed by Saaty (Saaty, 1980) is widely used, despite the criticism (Holder, 1990), (Elliott, 2010). Several alternative scales have been suggested (Ji \& Jiang, 2002), (Yucheng, Yinfeng, \& Hongyi, 2008), however, all of them are multiplicative scales. In this paper we define parameterized scales and move away from multiplicativity.

The structure of the article as follows. Section 2 reviews two approaches to the eigenvector method. In the third section two non-multiplicative scales are presented. The forth section describes two simulation studies carried out to compare presented scales with the fundamental one. In the fifth section two kinds of inconsistency are considered and two consistency measures are presented and compared via a simulation study. The final section summaries results of the paper.

\section{Two approaches to the eigenvector method}

Let us have a set of $n$ objects $C_{1}, C_{2}, \ldots, C_{n}$ that are compared to each other. We define a pairwise comparison matrix $A=\boldsymbol{\bigotimes}_{i j}{ }_{i=1}^{n}$, where $a_{i j}$ is a numerical estimate of the pair $\boldsymbol{C}_{i}, C_{j}$. The eigenvector method assumes that relative priorities of the objects $C_{1}, C_{2}, \ldots, C_{n}$ are equal to components of the eigenvector that corresponds to the maximum eigenvalue of the matrix $A$. Perron-Frobenius theorem claims that there is one and only one eigenvector with positive components if every element of the matrix $A$ is posi-

${ }^{*}$ Corresponding author 
tive. There are two approaches to the eigenvector method: one proposed by Saaty (Saaty, 1980) and the other proposed by Berge (Berge, 1962).

\subsection{Saaty approach of the eigenvector method}

The approach suggested by Saaty is based on the assumption that there are actual weights $w_{1}, w_{2}, \ldots, w_{n}$ of the objects $C_{1}, C_{2} \ldots, C_{n}$, and estimates $a_{i j}$ of pairs $\boldsymbol{C}_{i}, C_{j}$, are approximations of the ratios $w_{i} / w_{j}$. If we assume that $a_{i j}=w_{i} / w_{j}$, the following matrix equation comes out right:

$$
A w=\left(\begin{array}{cccc}
1 & w_{1} / w_{2} & \cdots & w_{1} / w_{n} \\
w_{2} / w_{1} & 1 & \cdots & w_{2} / w_{n} \\
\vdots & \vdots & \ddots & \vdots \\
w_{n} / w_{1} & w_{n} / w_{2} & \cdots & 1
\end{array}\right)\left(\begin{array}{c}
w_{1} \\
w_{2} \\
\vdots \\
w_{n}
\end{array}\right)=n w
$$

The equation $a_{i j}=w_{i} / w_{j}$ is not usually satisfied due to uncertainty and hesitations of a decision maker's judgments about pairs $\widehat{C}_{i}, C_{j}$. However, it is proved (Saaty, 1980) that components of the principal eigenvector are stable when elements $a_{i j}$ of the matrix $A$ are slightly disturbed from the values $w_{i} / w_{j}$.

\subsection{Berge's approach of the eigenvector method}

The approach suggested by Berge is based on the notion of an iterated power. According to Berge, the iterated power of the order $k$ of the object $C_{i}$ is the value $p^{i}(k)=p_{1}^{i}(k)+p_{2}^{i}(k)+\cdots+p_{n}^{i}(k)$, where $p_{j}^{i}(k)$ is the element in the row $i$ and the column $j$ of the matrix $A^{k}$. With $k$ increasing, the values of the iterated power of the order $k$ approach to the components of the principal eigenvector of the matrix $A$. So, unlike approach of Saaty, this approach does not require any assumption about estimates of pairwise comparisons.

\section{Alternative scales definition}

After a decision maker finishes comparing objects, the qualitative estimates he/she has made are converted into numerical ones. Let us associate qualitative estimates of relative importance with integer numbers. The higher the degree of superiority of an object in a pair upon another one is, the greater this number will be. Zero will be associated with equivalence of objects in a pair. Also positive numbers will correspond to superiority of the first element in a pair, the negative - to superiority of the second one. Let us designate the set of numbers corresponding to the qualitative estimates as $\Lambda=\ldots,-8,-7, \ldots,-1,0,1, \ldots, 7,8, \ldots$. According to (Ji \& Jiang, 2002), $\Lambda$ is a verbal part of a scale. The verbal part will be common for every concerned scale.

Now we define numerical parts of scales (Ji \& Jiang, 2002) as functions that map $\Lambda$ to $\mathbf{R}(\lambda \in \Lambda)$.

Table 1. The functions that define numerical parts of the scales.

\begin{tabular}{|l|c|l|}
\hline Scale name & Function that defines the numerical part & Scale parameters \\
\hline Saaty scale & $\varphi_{S}(\lambda)=x_{S}|\lambda|+1$ & $x_{S}$ - scale parameter \\
\hline Bruck scale & $\varphi_{B}\left(\mp c_{B}+\lambda x_{B}\right.$ & $\begin{array}{l}c_{B} \text { - center parameter } \\
x_{B} \text { - scale parameter }\end{array}$ \\
\hline
\end{tabular}




\begin{tabular}{|l|l|l|}
\hline Logistic scale & $\varphi_{L} \ell \neq 2 /(+\exp \leftarrow \mu \lambda)$ & $\mu$ - steepness parameter \\
\hline
\end{tabular}

Saaty scale presented in the table 1 is a generalized version of the fundamental one $\left(x_{S}=1\right)$. Bruck has proposed his scale in (Bruck \& Burkov, 1972). A logistic scale has been introduced in (Sushkov \& Kusherbaeva, 2010) and has a property based on the assumption that there is a boundary between qualitative estimates after which the difference between the estimates becomes inessential.

\section{Statistical study of the scales}

Let us introduce the following theorem which will help us to understand the statistical results below. Due to the limited size of the paper the proof of the theorem that has been presented in (Kusherbaeva \& Sushkov, 2010) is omitted.

Theorem. Let $A$ be a pairwise comparison matrix of $n$ alternatives, $w$ - the vector of priorities gained by the eigenvector method. The following statements come out right:

For Bruck scale:

- for every $c_{B}, x_{B}$ satisfying $c_{B} / x_{B}=$ const we have the invariable $w$;

- $\lim _{c_{B} \rightarrow \infty} w=1 / n$ when $x_{B}$ is fixed; $\lim _{x_{B} \rightarrow 0} w=1 / n$ when $c_{B}$ is fixed.

For Saaty scale:

- $\quad$ when multiplying $x_{S}$ by a positive number, $w$ does not change;

- $\lim _{x_{s} \rightarrow \infty} w_{i}=\left\{\begin{array}{l}1 / k \text { for the alternatives with maximal priority, } \\ \text { where } k \text { is the number of such alternatives; } \\ 0 \text { for other alternatives. }\end{array}\right.$

For the logistic scale:

- with $\mu$ increasing, the weights of the alternatives approach some limited values. The result will coincide with the case of Bruck scale with two grades on it,

- $\lim _{\mu \rightarrow 0} w_{i}=1 / n$.

Let us make some definitions (Kusherbaeva \& Sushkov, 2010).

Def. 1. If an element $A$ overcomes an element $B$, we will denote it in the following way: $A \succ B$, if $B$ overcomes $A: B \succ A$, if $A$ and $B$ are equal: $A \sim B$. Also, if there is a quantitative characteristic $m$ which describes how much an element overcomes another one, we will denote it as $A \stackrel{m}{\succ} B$.

Def. 2. Let $A$ be the $n \times n$ matrix of pairwise comparisons of objects $C_{1}, C_{2}, \ldots, C_{n}$, then $A$ satisfies the ordinal consistency, if $C_{i} \succ C_{j}, C_{j} \succ C_{k}$ involves $C_{i} \succ C_{k}$ for every $i, j, k$.

Let us describe cases of possible estimates:

Case 1. Let $C_{i} \stackrel{m}{\succ} C_{j} \stackrel{n}{\succ} C_{k}$ be objects. If $C_{i} \stackrel{l}{\succ} C_{k}$, and $l \geq \max (m, n), m, n \geq 0, \in \Lambda$, then the ordinal consistency is satisfied.

Remark. In this case for every scale under consideration (generally for every scale presented by a nondescending function) the resulting order of the alternatives will be the same. 
Case 2. Let $C_{i} \stackrel{m}{\succ} C_{j} \stackrel{n}{\succ} C_{k}$ be objects, $l, m, n \geq 0, \in \Lambda$. If $C_{i} \stackrel{l}{\succ} C_{k}$ and $0 \leq l \leq \max (m, n)$, then the resulting order can change depending on the scale being used.

Case 3. Let $C_{i} \stackrel{m}{\succ} C_{j} \stackrel{n}{\succ} C_{k}$ be objects $(m, n \geq 0, \in \Lambda)$. If $C_{k} \stackrel{l}{\succ} C_{i} l>0$, then the ordinal consistency does not take place and here we have a cycle; the resulting order can change depending on the cycle, the scale, and its parameters.

Experiment 1. Let us describe the first statistical experiment. Its goal is to investigate variances of priorities derived from the disturbed pairwise comparisons matrix depending on the scale being used. Suppose that there are seven objects estimated in the following way: $C_{1} \stackrel{1}{\succ}^{\prime} C_{2} \succ \ldots \stackrel{1}{\succ} C_{7}$. Estimates of pairwise comparisons between non-adjacent objects are generated according to the case 2 and for $\Lambda=\{-10 \ldots 10\}$. Then the matrix of qualitative pairwise comparisons $\Lambda=\left(\lambda_{i j}\right)_{i j=1}^{n}$ is built and fixed during the experiment. The disturbance matrix $\mathrm{E}=\left(\varepsilon_{i j}\right)_{i j=1}^{n}$ consists of independent realizations of the variate uniformly distributed on the set $-1,0,1$. During the experiment matrices $E$ are generated, the scale is applied to the elements of the matrix $\Lambda+\mathrm{E}$ and the priority vector is derived with the eigenvector method.

A sample of 10000 random matrices has been analyzed and results are the following. For Saaty scale with $x_{S}$ getting greater sample variances of priorities grow, and the higher the priority of the object is, the faster its sample variance grows. For Bruck scale if $x_{B}$ is fixed, the greater $c_{B}$ is, the less sample variances of the priorities are and vice versa: if $c_{B}$ is fixed, the greater $x_{B}$ is, the larger sample variances of the priorities are. Moreover, the sample variances are equal for every object being compared. For the logistic scale sample variances of the priorities of the objects that have the greatest priority value grow up to a limited value. It is caused by the special form of the scale. Sample variances of other objects behave in different ways depending on the pairwise comparison matrix.

To sum up, when having an error in a decision maker's conclusions, Saaty scale yields the greatest values of sample variances of the priorities, Bruck scale - the least values of the sample variances, the logistic scale - intermediate values close to zero compared with the values from Saaty scale.

Experiment 2. The second experiment is aimed at investigating the stability of ranking depending on the scale being used. Suppose that there are five objects $C_{1} \prec \ldots \prec C_{5}$. Let us generate and fix the estimates of qualitative pairwise comparisons between adjacent objects as independent variates uniformly distributed on the integers from 1 to 10. Estimates between non-adjacent objects are generated in the same way as in the first experiment. Thus, for every initial order we have a set of pairwise comparison matrices. Scales with different parameters are applied to the matrix; the priority vector is derived with the eigenvector method and analyzed whether the ranking of objects coincides with the initial one.

A sample of 10000 random matrices has been analyzed and results are the following. For Saaty scale: the larger $x_{S}$ is the greater probability of coincidence for every object is, because the greatest priority approaches 1 and other priorities approach 0 . It makes the spread between the object with the greatest priority and other objects grow. For Bruck scale: having one parameter $x_{B}$ or $c_{B}$ fixed and varying the other one probabilities of coincidence almost do not change. For the logistic scale: when $\mu$ grows probabilities of coincidences of the objects also get larger. The reason is that quantitative estimates of pairwise com- 
parisons between the objects get equal to the same number, although qualitative estimates of the comparisons might have been different.

Thus, the best scale according to the criterion is the logistic scale, Bruck scale is the worst and Saaty scale is in the middle.

\section{Consistency measures}

In the original version of the analytic hierarchy process a pairwise comparison matrix $A=\mathbf{Q}_{i j}{ }_{i=1}^{n}$ is considered to be perfectly consistent if $a_{i k}=a_{i j} a_{j k}$ for every $i, j, k$ (Saaty, 1980). Considering the multiplicativity of the fundamental scale it means that elements of the perfectly consistent matrix are ratios of the actual weights $w_{1}, w_{2}, \ldots, w_{n}: a_{i j} a_{j k}=w_{i} / w_{j} \cdot w_{j} / w_{k}=w_{i} / w_{k}=a_{i k}$. It is proved by Saaty that if the matrix $A$ is perfectly consistent, then its principal eigenvalue is equal to the size of $A$, otherwise it is greater than the matrix size and is stable when elements $a_{i j}$ of the matrix $A$ are slightly disturbed from the values $w_{i} / w_{j}$. Basing on this property, Saaty defines a consistency index (CI) of the matrix $A$ in the following way: $C I=\boldsymbol{Q}_{\max }-n \geq\left(-1\right.$, where $\lambda_{\max }$ is the principal eigenvalue of the matrix $A$ and $n$ is its size.

Let $C_{1}, C_{2}, \ldots, C_{n}$ be a set of objects without equivalent ones and $A$ is a pairwise comparison matrix corresponding to these objects. Following (Gass, 1998) we define a matrix $B=\boldsymbol{Q}_{i j}^{n_{i=1}^{n}}$ that is based on $A$ in the following way: if $a_{i j} \geq 0$, then $b_{i j}=1$, otherwise $b_{i j}=0$. The matrix $B$ can be considered as an adjacency matrix of the directed graph and cycles of the graph will correspond to violations of the ordinal consistency in the matrix A. Further, we will consider circular triads. In (Kendall \& Smith, 1940) authors prove that a number of circular triads $c$ in the completed directed graph without multiple edges can be obtained by the formula $c=n(n-1)(n-2) / 6-\frac{1}{2} \sum_{i=1}^{n} s_{i}\left(s_{i}-1\right)$, where $n$ is the size of the matrix $B$ and $s_{i}$ is a sum of the elements of the $i$-th row of the matrix $B$. Also a formula for the maximum number of circular triads in the tournament graph with $n$ vertices is provided: $n\left(n^{2}-4\right)$ for $n$ even and $n\left(n^{2}-1\right)$ for $n$ odd. Basing on these two formulas, Kendall and Smith define a coefficient of consistency $\zeta$ in the following way: $\zeta=1-24 / C \zeta^{2}-1$ for $n$ odd, $\zeta=1-24 c / C \zeta^{2}-4$ for $n$ even. As it follows from the definition, $\zeta \in \prod, 1_{-}^{-}$and if there are no cyclic triads, then $\zeta=1$, and when increasing the number of cyclic triads $\zeta$ approaches zero.

Saaty consistency index looses its interpretation when either Bruck scale or the logistic one is used, because the scales are not multiplicative. Instead of CI it is possible to use a coefficient of consistency. To investigate the correlation between CI and $\zeta$ the statistical study has been carried out. Six samples of 100000 random pairwise comparison matrices with elements from the fundamental scale had been generated for each matrix size from 4 to 9 . Sample correlations between CI and $\zeta$ were calculated.

Table 2. Sample correlations between CI and $\zeta$.

\begin{tabular}{|l|l|l|l|l|l|l|}
\hline Matrix size & 4 & 5 & 6 & 7 & 8 & 9 \\
\hline Correlation & -0.8330 & -0.7789 & -0.7269 & -0.6847 & -0.6463 & -0.6209 \\
\hline
\end{tabular}


As we can see from the table 2, there is a connection between CI and $\zeta$, but it gets weaker when matrix size grows. We suppose that this effect can be explained by the ordinal essence of $\zeta$. There is no difference for $\zeta$ between the following two situations: $a_{i j}=2, a_{j k}=2, a_{i k}=5$ and $a_{i j}=2, a_{j k}=2, a_{i k}=4$, because there is no circular triad in both cases. On the other hand, CI does not recognize circular triads and other cycles. Instead of that CI measures the deviation of the pairwise comparison matrix from the matrix that consists of ratios of actual weights of the objects being compared.

\section{Conclusion}

In this paper we have defined two alternative non-multiplicative scales and compared them to the fundamental scale that is usually used in the AHP. Also the alternative consistency measure has been described and its correlation with the consistency index that is used with the fundamental scale has been investigated. Concepts that are introduced in the paper have been implemented in the software package - a decision making support system created by the authors (Kusherbaeva, Sushkov \& Tamazyan, 2011).

\section{REFERENCES}

Berge, C. (1962). The Theory of graphs and its applications. Menthuen.

Bruck, B.N., \& Burkov V.N. (1972). Methods of expert estimation in objects ranging problems (in Russian). Journal of the Academy of Sciences of the USSR. Engineering Cybernetics, 3, 29-39.

Elliott, M.A. (2010). Selecting numerical scales for pairwise comparisons. Reliability Engineering and System Safety, 95, 750-763.

Gass, S.I. (1998). Tournaments, transitivity and pairwise comparison matrices. Journal of the Operational Research Society, 49, 616-624.

Holder, R.D. (1990). Some Comments on the Analytic Hierarchy Process. Journal of the Operational Research Society, 41, 1073-1076.

Ji, P., \& Jiang, R. (2002). Scale transitivity in the AHP. Journal of the Operational Research Society, 54, 896-905.

Kendall, M., \& Smith, B. (1940). On the method of pair comparisons. Biometrika, 31, 324-345.

Kusherbaeva, V.T., \& Sushkov, Yu.A. (2010). Scales and their properties in the AHP (in Russian). Journal of the KBSC of the Academy of Sciences of Russia, 37.

Kusherbaeva, V., Sushkov Yu, \& Tamazyan G. (2011). AHPManager - a decision making support system based on the AHP. Proceedings of the 11th ISAHP; Italy, Sorrento, June 15-18, 2011.

Saaty, T.L. (1980). The analytic hierarchy process. McGraw-Hill.

Yucheng, D., Yinfeng, X., Hongyi, L., \& Min, D. (2008). A comparative study of the numerical scales and the prioritization method in the AHP. European Journal of the Operational Research, 186, 229-242. 